\title{
ON THE COVERING RADIUS OF CODES OVER Z $Z_{4}$ WITH CHINESE EUCLIDEAN WEIGHT
}

\author{
P. Chella Pandian ${ }^{1}$ and C. Durairajan ${ }^{2}$ \\ ${ }^{1}$ Department of Mathematics Srimad Andavan Arts and Science College(Autonomous), \\ India. \\ ${ }^{2}$ Department of Mathematics, Bharathidasan University, India.
}

\begin{abstract}
In this paper, we give lower and upper bounds on the covering radius of codes over the ring $Z_{4}$ with respect to chinese euclidean distance. We also determine the covering radius of various Repetition codes, Simplex codes Type $\alpha$ and Type $\beta$ and give bounds on the covering radius for MacDonald codes of both types over $Z_{4}$.
\end{abstract}

\section{KEYWORDS}

Covering radius, Codes over finite rings, Simplex codes, Hamming codes.

(2010) Mathematical Subject Classification: 94B25, 94B05, 11H31, $11 \mathrm{H} 71$.

\section{INTRODUCTION}

In the last decade, there are many researchers doing research on code over finite rings. In particular, codes over $Z_{4}$ received much attention $[1,2,3,7,9,13,14]$. The covering radius of binary linear codes were studied $[4,5]$. Recently the covering radius of codes over $\mathrm{Z}_{4}$ has been investigated with respect to chinese euclidean distances [10]. In 1999, Sole et al gave many upper and lower bounds on the covering radius of a code over $\mathrm{Z}_{4}$ with chinese euclidean distances. In [12], the covering radius of some particular codes over $Z_{4}$ have been investigated. In this correspondence, we consider the ring $Z_{4}$. In this paper, we investigate the covering radius of the Simplex codes of both types and MacDonald codes and repetition codes over $\mathrm{Z}_{4}$. We also generalized some of the known bounds in [1].

A linear code $\mathrm{C}$ of length $\mathrm{n}$ over $\mathrm{Z}_{4}$ is an additive subgroup of $Z_{4}^{n}$. An element of $\mathrm{C}$ is called a codeword of $C$ and a generator matrix of $C$ is a matrix whose rows generate $C$. In [10], the chinese Euclidean weight $\mathrm{w}_{\mathrm{CE}}(\mathrm{x})$ of a vector $\mathrm{x}$ is $\sum_{i=1}^{n}\left\{2-2 \cos \left(\frac{2 \pi x_{i}}{4}\right)\right\}$

A linear Gray map $\varphi$ from $Z_{4} \rightarrow Z_{2}^{2}$ is defined by $\varphi(x+2 y)=(y, x+y)$, for all $x+2 y \in Z_{4}$. The image $\varphi(C)$, of a linear code $C$ over $Z_{4}$ of length $n$ by the Gray map, is a binary code of length $2 n$ with same cardinality [13]. 
Any linear code $\mathrm{C}$ over $\mathrm{Z}_{4}$ is equivalent to a code with generator matrix $\mathrm{G}$ of the form

$$
G=\left[\begin{array}{ccc}
I_{k_{0}} & A & B \\
0 & 2 I_{k_{1}} & 2 D
\end{array}\right],
$$

where $A, B$ and $D$ are matrices over $Z_{4}$. Then the code $C$ contain all code words $\left[v_{0}, v_{1}\right] G$, where $v_{0}$ is a vector of length $k_{1}$ over $Z_{4}$ and $v_{1}$ is a vector of length $k_{2}$ over $Z_{2}$. Thus $C$ contains a total of $4^{k_{1}} 2^{k_{2}}$ codewords. The parameters of $\mathrm{C}$ are given [n, $4^{k_{1}} 2^{k_{2}}$, d] where $\mathrm{d}$ represents the minimum chinese Euclidean distance of $\mathrm{C}$.

A linear code $\mathrm{C}$ over $\mathrm{Z}_{4}$ of length $\mathrm{n}$, 2-dimension $\mathrm{k}$, minimum chinese euclidean distance $\mathrm{d}_{\mathrm{CE}}$ is called an $\left[\mathrm{n}, \mathrm{k}, \mathrm{d}_{\mathrm{CE}}\right]$ or simply an $[\mathrm{n}, \mathrm{k}]$ code.

In this paper, we define the covering radius of codes over $\mathrm{Z}_{4}$ with respect to chinese euclidean distance and in particular study the covering radius of Simplex codes of type $\alpha$ and type $\beta$ namely, $\mathrm{S}_{k}^{\alpha}$ and $\mathrm{S}_{k}^{\beta}$ and their MacDonald codes and repetition codes over $\mathrm{Z}_{4}$. Section 2 contains basic results for the covering radius of codes over $\mathrm{Z}_{4}$. Section 3 determines the covering radius of different types of repetition codes. Section 4 determines the covering radius of Simplex codes and finally section 5 determines the bounds on the covering radius of MacDonald codes.

\section{Covering Radius of Codes}

Let $\mathrm{d}$ be a chinese euclidean distance. The covering radius of a code $\mathrm{C}$ over $\mathrm{Z}_{4}$ with respect to chinese euclidean distance $\mathrm{d}$ is given by

$$
r_{d}(C)=\max _{u \in z_{4}^{n}}\left\{\min _{c \in C}\{d(c, u)\}\right\}
$$

The following result of Mattson [4] is useful for computing covering radius of codes over rings generalized easily from codes over finite fields.

Proposition 2. 5. (Mattson) If $\mathrm{C}_{0}$ and $\mathrm{C}_{1}$ are codes over $\mathrm{Z}_{4}$ generated by matrices $\mathrm{G}_{0}$ and $\mathrm{G}_{1}$ respectively and if $\mathrm{C}$ is the code generated by $\quad G=\left(\begin{array}{c|c}0 & G_{1} \\ \hline G_{0} & A\end{array}\right)$ then $\mathrm{r}_{\mathrm{d}}(\mathrm{C}) \leq \mathrm{r}_{\mathrm{d}}\left(\mathrm{C}_{0}\right)+\mathrm{r}_{\mathrm{d}}\left(\mathrm{C}_{1}\right)$ and the covering radius of $D$ (concatenation of $C_{0}$ and $C_{1}$ ) satisfy the following $r_{d}(D) \geq r_{d}\left(C_{0}\right)+$ $r_{d}\left(C_{1}\right)$, for all distances d over $Z_{4}$.

\section{COVERING RADIUS OF REPETITION CODES}

A q-ary repetition code $\mathrm{C}$ over a finite field $\mathrm{F}_{\mathrm{q}}=\left\{\alpha_{0}=0, \alpha_{1}=1, \alpha_{2}, \alpha_{3}, \ldots, \alpha_{\mathrm{q}-1}\right\}$ is an $[\mathrm{n}, 1, \mathrm{n}]$ code $C=\left\{\bar{\alpha} \mid \alpha \in F_{q}\right\}$, where $\bar{\alpha}=(\alpha, \alpha, \cdots, \alpha)$. The covering radius of $\mathrm{C}$ is $\left\lceil\frac{n(q-1)}{q}\right\rceil$ [11]. Using this, it can be seen easily that the covering radius of block of size $\mathrm{n}$ repetition code $[\mathrm{n}(\mathrm{q}-1), 1, \mathrm{n}(\mathrm{q}-1)]$ generated by $\quad G=[\overbrace{11 \cdots 1}^{n} \overbrace{\alpha_{2} \alpha_{2} \cdots \alpha_{2}}^{n} \overbrace{\alpha_{3} \alpha_{3} \cdots \alpha_{3} \cdots \alpha_{\alpha_{-1} \alpha_{q-1} \cdots \alpha_{q-1}}^{n}}^{n}]$ is 
International Journal on Information Theory (IJIT), Vol.4, No.4, October 2015

$\left\lceil\frac{n(q-1)^{2}}{q}\right\rceil$ since it will be equivalent to a repetition code of length $(\mathrm{q}-1) \mathrm{n}$.

Consider the repetition code over $\mathrm{Z}_{4}$. There are two types of them of length $\mathrm{n}$ viz. unit repetition code $\mathrm{C}_{\beta}:[\mathrm{n}, 1,2 \mathrm{n}]$ generated by $G_{\beta}=[\overbrace{11 \cdots 1}^{n}]$ and zero divisor repetition code $\mathrm{C}_{\alpha}:(\mathrm{n}, 2,4 \mathrm{n})$ generated by $G_{\alpha}=[\overbrace{22 \cdots 2}^{n}]$. The following result determines the covering radius with respect to chinese euclidean distance.

Theorem 3. 1. $4\left\lfloor\frac{n}{2}\right\rfloor \leq r_{C E}\left(C_{\alpha}\right) \leq 2 n$ and $r_{C E}\left(C_{\beta}\right)=2 n$.

$$
\overbrace{}^{\left\lfloor\frac{n}{2}\right\rfloor} \overbrace{}^{\left\lceil\frac{n}{2}\right\rceil}
$$

Proof. Let $\mathrm{x}=\overbrace{22 \cdots 200 \cdots 0} \in Z_{4}^{n}$. The code $C_{\alpha}=\left\{\alpha(22 \cdots 2) \mid \alpha \in Z_{4}^{n}\right\}$, that is $C_{\alpha}=$ $\{00 \ldots . .22 \ldots .2\}$, generated by $[22 \ldots .2]$ is an $[\mathrm{n}, 1,2 \mathrm{n}]$ code. Then, $\mathrm{d}_{\mathrm{CE}}(\mathrm{x}, 00 \ldots 0)=$ $\mathrm{wt}_{\mathrm{CE}}(\overbrace{22 \cdots 200 \cdots 0}^{\left\lfloor\frac{n}{2}\right\rfloor}-00 \cdots 0)=4\left\lceil\frac{n}{2}\right\rceil$ and $\mathrm{d}_{\mathrm{CE}}(\mathrm{x}, 22 \ldots .2)=$ $\mathrm{Wt}_{\mathrm{CE}}(\overbrace{22 \cdots 200 \cdots 0}^{\left\lfloor\frac{n}{2}\right\rfloor}-22 \cdots 2)=4\left\lfloor\frac{n}{2}\right\rfloor$. Therefore $\mathrm{d}_{\mathrm{CE}}\left(\mathrm{x}, C_{\alpha}\right)=\min \left\{4\left\lceil\frac{n}{2}\right\rceil, 4\left\lfloor\frac{n}{2}\right\rfloor\right\}$.

Thus, by definition of covering radius $\mathrm{r}_{\mathrm{CE}}\left(C_{\alpha}\right) \geq 4\left\lfloor\frac{n}{2}\right\rfloor$

Let $\mathrm{x}$ be any word in $Z_{4}^{n}$. Let us take $\mathrm{x}$ has $\omega_{0}$ coordinates as 0 's, $\omega_{1}$ coordinates as 1 's, $\omega_{2}$ coordinates as 2 's, $\omega_{3}$ coordinates as 3's, then $\omega_{0+} \omega_{1+}, \omega_{2+} \omega_{3}=\mathrm{n}$. Since $C_{\alpha}=\{00 \ldots 0,22 \ldots 2\}$ and chinese euclidean weight of $Z_{4}$ : 0 is 0,1 and 3 is 2 and 2 is 4 , we have $d_{C E}(x, 00 \ldots 0)=n-\omega_{0}$ $+\omega_{1}+3 \omega_{2+} \omega_{3}$ and $d_{\mathrm{CE}}(\mathrm{x}, 22 \ldots 2)=\mathrm{n}-\omega_{2}+\omega_{1}+3 \omega_{0+} \omega_{3}$.

Thus $d_{C E}\left(x, C_{\alpha}\right)=\min \left\{n-\omega_{0}+\omega_{1}+3 \omega_{2+} \omega_{3}, n-\omega_{2}+\omega_{1}+3 \omega_{0+} \omega_{3}\right\}$.

$$
\mathrm{d}_{\mathrm{CE}}\left(\mathrm{x}, \mathrm{C}_{\alpha}\right) \leq \mathrm{n}++\mathrm{n}=2 \mathrm{n}
$$

Hence, from the Equation (3.1) and (3.2), we get $4\left\lfloor\frac{n}{2}\right\rfloor \leq r_{C E}\left(C_{\alpha}\right) \leq 2 n$. Now, we find the covering radius of $\mathrm{C}_{\beta}$ covering with respect to the chinese euclidean weight. We have $\mathrm{d}_{\mathrm{CE}}(\mathrm{x}, 00 \ldots 0)=\mathrm{n}-\omega_{0}+\omega_{1}+3 \omega_{2+} \omega_{3,} \mathrm{~d}_{\mathrm{CE}}(\mathrm{x}, 11 \ldots 1)=\mathrm{n}-\omega_{1}+\omega_{0}+\omega_{2+} 3 \omega_{3,} \mathrm{~d}_{\mathrm{CE}}(\mathrm{x}, 22 \ldots 2)=\mathrm{n}-$ $\omega_{2}+3 \omega_{1}+\omega_{3}$ and $\mathrm{d}_{\mathrm{CE}}(\mathrm{x}, 33 \ldots 3)=\mathrm{n}-\omega_{3}+3 \omega_{1}+\omega_{0+} \omega_{2}$ for any $x \in Z_{4}^{n}$. This implies $\mathrm{d}_{\mathrm{CE}}(\mathrm{x}$, $\left.\mathrm{C}_{\beta}\right)=\min \left\{\mathrm{n}-\omega_{0}+\omega_{1}+3 \omega_{2+} \omega_{3,} \mathrm{n}-\omega_{1}+\omega_{0}+\omega_{2+} 3 \omega_{3,} \mathrm{n}-\omega_{2}+3 \omega_{1}+\omega_{3}, \mathrm{n}-\omega_{3}+3 \omega_{1}+\omega_{0+}\right.$ $\left.\omega_{2}\right\} \leq 2 \mathrm{n}$ and hence $\mathrm{r}_{\mathrm{CE}}\left(\mathrm{C}_{\beta}\right) \leq 2 \mathrm{n}$. To show that $\mathrm{r}_{\mathrm{CE}}\left(\mathrm{C}_{\beta}\right) \geq 2 \mathrm{n}$, let 
$x=\overbrace{00 \cdots 011 \cdots 122 \cdots 233 \cdots 3}^{t} \in \overbrace{Z_{4}^{n}}^{t} \overbrace{}^{n-3 t}$, where $\mathrm{t} \quad=\left\lfloor\frac{n}{4}\right\rfloor, \quad$ then $\mathrm{d}_{\mathrm{CE}}(\mathrm{x}, 00 \ldots 0)=2 \mathrm{n}$, $\mathrm{d}_{\mathrm{CE}}(\mathrm{x}, 11 \ldots 1)=4 \mathrm{n}-8 \mathrm{t}, \mathrm{d}_{\mathrm{CE}}(\mathrm{x}, 22 \ldots 2)=2 \mathrm{n}$ and $\mathrm{d}_{\mathrm{CE}}(\mathrm{x}, 33 \ldots . .3)=8 \mathrm{t}$.

Therefore $\mathrm{r}_{\mathrm{CE}}\left(\mathrm{C}_{\beta}\right) \geq \min \{2 \mathrm{n}, 4 \mathrm{n}-8 \mathrm{t}, 8 \mathrm{t}\} \geq 2 \mathrm{n}$.

To determine the covering radius of $\mathrm{Z}_{4}$ three blocks each of size $\mathrm{n}$ repetition code BRep ${ }^{3 n}:[3 \mathrm{n}$, $1,8 \mathrm{n}]$ generated by $G=[\overbrace{11 \cdots 122 \cdots 233 \cdots 3}^{n}]$ note that the code has constant chinese euclidean weight $8 \mathrm{n}$ and the block repetition code BRep ${ }^{3 n}:\left\{\mathrm{c}_{0}=\left(\begin{array}{lllll}0 \ldots 0 & 0 \ldots .0 & 0 \ldots . .0\end{array}\right), \mathrm{c}_{1}=\left(\begin{array}{ll}1 \ldots .12 \ldots .2 \\ 0.12\end{array}\right.\right.$ $3 \ldots .3), \mathrm{c}_{2}=\left(\begin{array}{lllll}2 \ldots 2 & 0 & \ldots & 2 & 2 \ldots 2\end{array}\right), \mathrm{c}_{3}=\left(\begin{array}{lllll}3 \ldots 3 & 2 \ldots .2 & 1 \ldots .1\end{array}\right)$. Thus $\mathrm{x}=11 \cdots 1 \in Z_{4}^{3 n}$, we have $\mathrm{d}_{\mathrm{CE}}$ $\left(\mathrm{x}_{\text {, BRep }}^{3 n}\right)=4\left\lfloor\frac{n}{2}\right\rfloor+4 n$. Hence by definition, $\mathrm{r}_{\mathrm{CE}}\left(\right.$ BRep $\left.^{3 n}\right) \geq 4\left\lfloor\frac{n}{2}\right\rfloor+4 n$. To find the upper Let $\mathrm{x}=(\mathrm{u}|\mathrm{v}| \mathrm{w}) \in Z_{4}^{3 n}$ with $\mathrm{u}, \mathrm{v}$ and $\mathrm{w}$ have compositions $\left(\mathrm{r}_{0}, \mathrm{r}_{1}, \mathrm{r}_{2}, \mathrm{r}_{3}\right),\left(\mathrm{s}_{0}, \mathrm{~s}_{1}, \mathrm{~s}_{2}\right.$, $\left.\mathrm{s}_{3}\right)$ and $\left(\mathrm{t}_{0}, \mathrm{t}_{1}, \mathrm{t}_{2}, \mathrm{t}_{3}\right)$ respectively such that $\sum_{i=0}^{3} r_{i}=n, \sum_{i=0}^{3} s_{i}=n$ and $\sum_{i=0}^{3} t_{i}=n$, then $\mathrm{d}_{\mathrm{CE}}$ $\left(x, c_{0}\right)=3 n-r_{0}+r_{1}+3 r_{2}+r_{3}-s_{0}+s_{1}+3 s_{2}+s_{3}-t_{0}+t_{1}+3 t_{2}+t_{3}, d_{C E}\left(x, c_{1}\right)=3 n-r_{1}+r 0+r 2+3 r_{3}$ $-\mathrm{s}_{2}+3 \mathrm{~s} 0+\mathrm{s} 1+\mathrm{s} 3_{0}-\mathrm{t}_{3}+\mathrm{t}_{0}+3 \mathrm{t}_{1}+\mathrm{t}_{2}, \mathrm{~d}_{\mathrm{CE}}\left(\mathrm{x}, \mathrm{c}_{2}\right)=3 \mathrm{n}-\mathrm{r}_{2}+\mathrm{r}_{1}+3 \mathrm{r}_{0}+\mathrm{r}_{3}-\mathrm{s}_{0}+\mathrm{s}_{1}+3 \mathrm{~s}_{2}+\mathrm{s}_{3}-\mathrm{t}_{2}+3 \mathrm{t}_{0}+\mathrm{t}_{1}+\mathrm{t}_{3}$ and $\mathrm{d}_{\mathrm{CE}}\left(\mathrm{x}, \mathrm{c}_{3}\right)=3 \mathrm{n}-\mathrm{r}_{3}+3 \mathrm{r} 1+\mathrm{r} 0+\mathrm{r} 2-\mathrm{s}_{2}+3 \mathrm{~s}_{0}+\mathrm{s}_{1}+\mathrm{s}_{3}-\mathrm{t}_{1}+3 \mathrm{t}_{3}+\mathrm{t}_{0}+\mathrm{t}_{2}$. Thus $\mathrm{d}_{\mathrm{CE}}\left(\mathrm{x}\right.$, BRep $\left.{ }^{3 n}\right)=$ $\min \left\{3 n-r_{0}+r_{1}+3 r_{2}+r_{3}-s_{0}+s_{1}+3 s_{2}+s_{3}-t_{0}+t_{1}+3 t_{2}+t_{3}, 3 n-r_{1}+r 0+r 2+3 r_{3}-s_{2}+3 s 0+s 1+s 3_{0}\right.$ $-\mathrm{t}_{3}+\mathrm{t}_{0}+3 \mathrm{t}_{1}+\mathrm{t}_{2}, 3 \mathrm{n}-\mathrm{r}_{2}+\mathrm{r}_{1}+3 \mathrm{r}_{0}+\mathrm{r}_{3}-\mathrm{s}_{0}+\mathrm{s}_{1}+3 \mathrm{~s}_{2}+\mathrm{s}_{3}-\mathrm{t}_{2}+3 \mathrm{t}_{0}+\mathrm{t}_{1}+\mathrm{t}_{3}, 3 \mathrm{n}-\mathrm{r}_{3}+3 \mathrm{r} 1+\mathrm{r} 0+\mathrm{r} 2$ $-\mathrm{s}_{2}+3 \mathrm{~s}_{0}+\mathrm{s}_{1}+\mathrm{s}_{3}-\mathrm{t}_{1}+3 \mathrm{t}_{3}+\mathrm{t}_{0}+\mathrm{t}_{2}$. Thus we have the following theorem

Theorem 3. 2. $4\left\lfloor\frac{n}{2}\right\rfloor+4 n \leq \mathrm{r}_{\mathrm{CE}}\left(\mathrm{BRep}^{3 n}\right) \leq 6 n$.

One can also define a $\mathrm{Z}_{4}$ block (two blocks each of size $\mathrm{n}$ ) repetition code Brep ${ }^{2 n}:[2 \mathrm{n}, 1,4 \mathrm{n}]$ generated by $G=[\overbrace{11 \cdots 122 \cdots 2}^{n} \overbrace{}^{n}$. We have following theorem

Theorem 3. 3. $4\left\lfloor\frac{n}{2}\right\rfloor+2 n \leq \mathrm{r}_{\mathrm{CE}}\left(\mathrm{BRep}^{3 n}\right) \leq 4 \mathrm{n}$

Block code BRep ${ }^{m+n}$ can be generalized to a block repetition code (two blocks of size $\mathrm{m}$ and $\mathrm{n}$

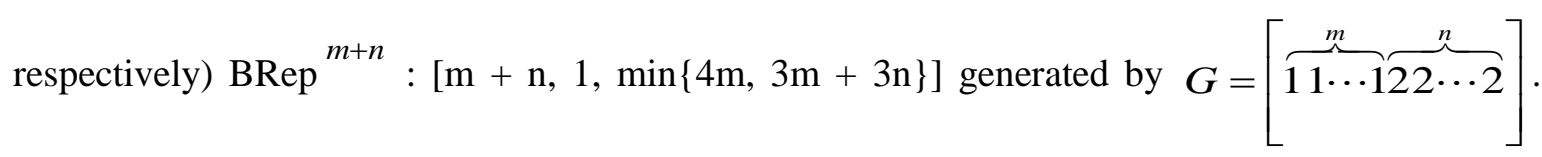
Theorem 3.3 can be easily generalized for different length using similar arguments to the following.

Theorem 3. 4. $2 m+4\left\lfloor\frac{n}{2}\right\rfloor \leq \mathrm{r}_{\mathrm{CE}}\left(\mathrm{BRep}^{m+n}\right) \leq 2 \mathrm{~m}+2 \mathrm{n}$ 


\section{SIMPLEX CODES OF TYPE A AND TYPE $\beta$ OVER $Z_{4}$}

Quaternary simplex codes of type $\alpha$ and type $\beta$ have been recently studied in [2]. Type $\alpha$ Simplex code $S_{k}^{\alpha}$ is a linear code over $\mathrm{Z}_{4}$ with parameters $\left[4^{\mathrm{k}}, \mathrm{k}\right]$ and an inductive generator matrix given by

$$
G_{k}^{\alpha}=\left[\begin{array}{c|c|c|c|c}
\text { OOO..OO } & 11 \ldots 1 & 22 \ldots 2 & 33 \ldots 3 \\
\hline G_{k-1}^{\alpha} & G_{k-1}^{\alpha} & G_{k-1}^{\alpha} & G_{k-1}^{\alpha}
\end{array}\right]
$$

with $G_{1}^{\alpha}=\left[\begin{array}{lll}0 & 1 & 2\end{array}\right]$. Type simplex code $S_{k}^{\beta}$ is a punctured version of $S_{k}^{\alpha}$ with parameters [2 $2^{\mathrm{k}-1}$ $\left.\left(2^{\mathrm{k}}-1\right), \mathrm{k}\right]$ and an inductive generator matrix given by

$$
G_{2}^{\beta}=\left[\begin{array}{llll|l|l}
1 & 1 & 1 & 1 & 0 & 2 \\
\hline 0 & 1 & 2 & 3 & 1 & 1
\end{array}\right]
$$

and for $k>2$

$$
G_{k}^{\beta}=\left[\begin{array}{c|c|c}
11 \cdots 1 & 00 \cdots 0 & 22 \cdots 2 \\
\hline G_{k-1}^{\alpha} & G_{k-1}^{\beta} & G_{k-1}^{\beta}
\end{array}\right]
$$

where $G_{k-1}^{\alpha}$ is the generator matrix of $S_{k-1}^{\alpha}$. For details the reader is refered to [2]. Type $\alpha$ code with minimum chinese euclidean weight is 8 .

Theorem 4. 1. $r_{\mathrm{CE}}\left(S_{k}^{\alpha}\right) \leq 2^{2 \mathrm{k}+1}-3$.

Proof. Let $x=11 \ldots \ldots 1 \in Z_{4}^{n}$. By equation 4.1, the result of Mattson for finite rings and using Theorem 3. 2, we get

$$
\begin{aligned}
r_{\mathrm{CE}}\left(S_{k}^{\alpha}\right) & \leq r_{\mathrm{CE}}\left(S_{k-1}^{\alpha}\right)+r_{\mathrm{CE}}(<\overbrace{11 \cdots 1}^{2^{2(k-1)}} \overbrace{22 \cdots 2}^{2^{2(k-1)}} \overbrace{33 \cdots 3}^{2^{2(k-1)}}>) \\
& =r_{\mathrm{CE}}\left(S_{k-1}^{\alpha}\right)+6.2^{2(\mathrm{k}-1)} \\
& =6.2^{2(\mathrm{k}-1)}+6.2^{2(\mathrm{k}-2)}+6.2^{2(\mathrm{k}-3)}+\ldots . .+6.2^{2.1}+r_{\mathrm{CE}}\left(S_{1}^{\alpha}\right) \\
r_{\mathrm{CE}}\left(S_{k}^{\alpha}\right) & \leq 2^{2 \mathrm{k}+1}-3\left(\text { since } r_{\mathrm{CE}}\left(S_{1}^{\alpha}\right)=5\right)
\end{aligned}
$$

Theorem 4. 2. $r_{\mathrm{CE}}\left(S_{k}^{\beta}\right) \leq 2^{\mathrm{k}}\left(2^{\mathrm{k}}-1\right)-7$

Proof. By equation 4. 3, Proposition 2. 5 and Theorem 3. 4, we get

$$
\begin{aligned}
r_{\mathrm{CE}}\left(S_{k}^{\beta}\right) & \leq r_{\mathrm{CE}}\left(S_{k-1}^{\beta}\right)+r_{\mathrm{CE}}(<\overbrace{11 \cdots 1}^{4^{(k-1)}} \overbrace{22 \cdots 2}^{2^{(2 k-3)}-2^{(k-2)}}>) \\
& =r_{\mathrm{CE}}\left(S_{k-1}^{\beta}\right)+2^{(2 \mathrm{k}-2)}+2^{(2 \mathrm{k}-3)}-2^{(\mathrm{k}-2)}
\end{aligned}
$$


International Journal on Information Theory (IJIT), Vol.4, No.4, October 2015

$$
\begin{aligned}
\leq & 2\left(2^{(2 \mathrm{k}-2)}+2^{(2 \mathrm{k}-4)}+\ldots+2^{4}\right)+2\left(2^{(2 \mathrm{k}-3)}+2^{(2 \mathrm{k}-5)}+\ldots+2^{3}\right)- \\
& 2\left(2^{(\mathrm{k}-2)}+2^{(\mathrm{k}-3)}+\ldots+2\right)+r_{\mathrm{CE}}\left(S_{2}^{\beta}\right)
\end{aligned}
$$

$r_{\mathrm{CE}}\left(S_{k}^{\beta}\right) \leq 2^{\mathrm{k}-1}\left(2^{\mathrm{k}}-1\right)-7\left(\right.$ since $\left.r_{\mathrm{CE}}\left(S_{2}^{\beta}\right)=5\right)$.

\section{MACDONALD CODES CODES OF TYPE A $A$ ND $\beta$ OVER $\mathrm{Z}_{4}$}

The $q$-ary MacDonald code $M_{k, t}(q)$ over the finite field $\mathrm{F}_{q}$ is a unique $\left[\frac{q^{k}-q^{t}}{q-1}, k, q^{k-1}-q^{t-1}\right]$ code in which every non-zero codeword has weight either $q^{k-1}$ or $q^{k-1}-q^{t-1}$ [8]. In [11], he studied the covering radius of MacDonald codes over a finite field. In fact, he has given many exact values for smaller dimension. In [6], authors have defined the MacDonald codes over a ring using the generator matrices of simplex codes. For $2 \leq t \leq k-1$, let $\boldsymbol{G}_{k, t}^{\alpha}$ be the matrix obtained from $\boldsymbol{G}_{k}^{\alpha}$, by deleting columns corresponding to the columns of $G_{t}^{\alpha}$. That is,

$$
G_{k, t}^{\alpha}=\left[G_{k}^{\alpha} \backslash \frac{0}{G_{t}^{\alpha}}\right]
$$

and let $G_{k, t}^{\beta}$ be the matrix obtained from $G_{k}^{\beta}$ by deleting columns corresponding to the columns of $G_{t}^{\beta}$. That is,

$$
G_{k, t}^{\beta}=\left[G_{k}^{\beta} \backslash \frac{0}{G_{t}^{\beta}}\right]
$$

where $[A \backslash B]$ denotes the matrix obtained from the matrix $A$ by deleting the columns of the matrix $B$ and $\mathbf{0}$ is a $(k-t) \times 2^{2 \mathrm{t}}\left((k-t) \times 2^{\mathrm{t}-1}\left(2^{\mathrm{t}}-1\right)\right)$. The code generated by the matrix $G_{k, t}^{\alpha}$ is called code of type $\alpha$ and the code generated by the matrix $G_{k, t}^{\beta}$ is called Macdonald code of type $\beta$. The type $\alpha$ code is denoted by $M_{k, t}^{\alpha}$ and the type $\beta$ code is denoted by $M_{k, t}^{\beta}$. The $M_{k, t}^{\alpha}$ code is $\left[4^{\mathrm{k}}-4^{\mathrm{t}}, \mathrm{k}\right]$ code over $\mathrm{Z}_{4}$ and $M_{k, t}^{\beta}$ is a $\left[\left(2^{\mathrm{k}-1}-2^{\mathrm{t}-1}\right)\left(2^{\mathrm{k}}+2^{\mathrm{t}}-1\right), \mathrm{k}\right]$ code over $\mathrm{Z}_{4}$. In fact, these codes are punctured code of $S_{k}^{\alpha}$ and $S_{k}^{\beta}$ respectively.

Next Theorem gives a basic bound on the covering radius of above Macdonald codes.

\section{Theorem 5. 1.}

$$
r_{C E}\left(M_{k, t}^{\alpha}\right) \leq 2^{2 k+1}-2^{2 r+1}+r_{C E}\left(M_{r, t}^{\alpha}\right) \text { for } \mathrm{t}<\mathrm{r} \leq \mathrm{k}
$$

Proof. By Proposition 2.1 and Theorem 3.2,

$$
r_{\mathrm{CE}}\left(\mathrm{M}_{k, t}^{\alpha}\right) \leq r_{\mathrm{CE}}(<\overbrace{11 \cdots 1}^{2^{2(k-1)}} \overbrace{22 \cdots 2}^{2^{2(k-1)}} \overbrace{33 \cdots 3}^{2^{2(k-1)}}>)+r_{\mathrm{CE}}\left(\mathrm{M}_{r, t}^{\alpha}\right)
$$


International Journal on Information Theory (IJIT), Vol.4, No.4, October 2015

$$
\begin{aligned}
& =6.4^{k-1}+r_{C E}\left(M_{k-1, t}^{\alpha}\right), \text { for } \mathrm{k} \geq \mathrm{r}>\mathrm{t} . \\
& \leq 6.4^{k-1}+6.4^{k-2}+\ldots .+6.4^{r}+r_{C E}\left(M_{r, t}^{\alpha}\right) \text { for } \mathrm{k} \geq \mathrm{r}
\end{aligned}
$$

$>\mathrm{t}$.

$$
r_{\mathrm{CE}}\left(\mathrm{M}_{k, t}^{\alpha}\right) \leq 2^{2 \mathrm{k}+1}-2^{2 \mathrm{r}+1}+r_{\mathrm{CE}}\left(\mathrm{M}_{r, t}^{\alpha}\right), \text { for } \mathrm{k} \geq \mathrm{r}>\mathrm{t} .
$$

Theorem 5. 2.

$$
r_{C E}\left(M_{k, t}^{\beta}\right) \leq 2^{k}\left(2^{k}-1\right)+2^{r}\left(1-2^{r}\right)+r_{C E}\left(M_{r, t}^{\beta}\right) \text { for } \mathrm{t}<\mathrm{r} \leq \mathrm{k} .
$$

Proof. Using Proposition 2.1 and Theorem 3.4, we have

$$
\begin{aligned}
& r_{C E}\left(M_{k, t}^{\beta}\right) \leq r_{\mathrm{CE}}(<\overbrace{11 \cdots 1}^{2^{2(k-1)}} \overbrace{22 \cdots 2}^{2^{2(k-1)-1}-2^{(k-1)-1}}>)+r_{C E}\left(M_{k-1, t}^{\beta}\right) \\
& \leq 2.22^{(\mathrm{k}-1)+2} 2 \cdot 2^{2(\mathrm{k}-1)-1}-2^{(\mathrm{k}-1)-1}+r_{C E}\left(M_{k-1, t}^{\beta}\right) \\
&=2.2^{2(\mathrm{k}-1)+} 2.2^{2(\mathrm{k}-1)-1}-2^{2(\mathrm{k}-1)-1}+2 \cdot 2^{2(\mathrm{k}-2)}+2.2^{2(\mathrm{k}-2)-1}-2.2^{2(\mathrm{k}-2)-1}+r_{C E}\left(M_{k-2, t}^{\beta}\right) \\
& \leq 2.2^{2(\mathrm{k}-1)+} 2.2^{2(\mathrm{k}-1)-1}-2^{2(\mathrm{k}-1)-1}+2.2^{2(\mathrm{k}-2)}+2.2^{2(\mathrm{k}-2)-1}-2.2^{2(\mathrm{k}-2)-1}+\ldots+ \\
& 2.2^{2 \mathrm{r}}+2.2^{2 \mathrm{r}-1}+2.2^{\mathrm{r}-1}+r_{C E}\left(M_{r, t}^{\beta}\right) \\
&=2^{2 \mathrm{k}}-2^{2 \mathrm{r}}-2^{\mathrm{k}}+2^{\mathrm{r}}+r_{C E}\left(M_{r, t}^{\beta}\right), \mathrm{t}<\mathrm{r} \leq \mathrm{k} . \\
& r_{C E}\left(M_{k, t}^{\beta}\right) \leq 2^{\mathrm{k}}\left(2^{\mathrm{k}}-1\right)+2^{\mathrm{r}}\left(1-2^{\mathrm{r}}\right)+r_{C E}\left(M_{r, t}^{\beta}\right), \mathrm{t}<\mathrm{r} \leq \mathrm{k} .
\end{aligned}
$$

\section{ACKNOWLEDGEMENTS}

The first author was supported by a grant (F. No.: 4-4/2014-15(MRP-SEM/UGC-SERO, Nov. 2014)) for the University Grants Commission, South Eastern Regional office, Hyderabad - 500 001 .

\section{References}

[1] Aoki T., Gaborit P., Harada, M., Ozeki, M. and Sol'e P., " On the covering radius of Z4 codes and their lattices", IEEE Trans. Inform. Theory, vol. 45, no. 6, pp. 2162-2168(1999).

[2] Bhandari M. C., Gupta M. K. and Lal A. K., " On Z4 Simplex codes and their gray images", Applied Algebra, Algebraic Algorithms and Error-Correcting Codes, AAECC- 13, Lecture Notes in Computer Science 1719, 170-180(1999) .

[3] Bonnecaze A., Sol'e, P., Bachoc, C. and Mourrain B., “ Type II codes over Z4”, IEEE Trans. Inform. Theory, 43, 969-976(1997).

[4] Cohen G. D., Karpovsky, M. G., Mattson, H. F. and Schatz J. R., “ Covering radius- Survey and recent results", IEEE Trans. Inform. Theory, vol. 31, no. 3, pp. 328-343(1985).

[5] Cohen C., Lobstein, A. and Sloane N. J. A., " Further Results on the Covering Radius of codes", IEEE Trans. Inform. Theory, vol. 32, no. 5, 1986, pp. 680-694(19977) .

[6] Colbourn, C. J. and Gupta M. K., “ On quaternary MacDonald codes”, Proc. Information Technology:Coding and Computing (ITCC), pp. 212-215 April(2003).

[7] Conway, J. H. and Sloane N. J. A., 1993, "Self-dual codes over the integers modulo 4", Journal of Combin. Theory Ser. A 62, 30-45(1993). 
International Journal on Information Theory (IJIT), Vol.4, No.4, October 2015

[8] Dodunekov S. and Simonis, J., "Codes and projective multisets", The Electronic Journal of Communications 5 R37(1998).

[9] Dougherty S. T., Harada M. and Sol'e P., “ Shadow codes over Z4”, Finite Fields and Their Appl. (to appear).

[10] Gupta M. K., David G. Glynn and Aaron Gulliver T., On Senary Simplex Codes. Lecture Notes in Computer Science.

[11] Durairajan C., “ On Covering Codes and Covering Radius of Some Optimal Codes”, Ph. D. Thesis, Department of Mathematics, IIT Kanpur (1996).

[12] Gupta M. K and Durairajan C., On the Covering Radius of some Modular Codes. Journal of Advances in Mathematics of Computations 8(2), 9, 2014.

[13] Hammons A. R., Kumar P. V., Calderbank A. R., Sloane N. J. A. and Sol'e P.,

The Z4-linearity of kerdock, preparata, goethals, and related codes.IEEE Trans. Inform. Theory, 40, 301-319 (1994).

[14] Harada M., New extremal Type II codes over Z4. Des. Codes and Cryptogr. 13, 271-284 (1998). 